\title{
Analysis on the solar irradiance fluctuations effect on the BepiColombo Superior Conjunction Experiment
}

\author{
Ivan di Stefano \\ Sapienza University of Rome, \\ Dipartimento di Ingegneria Meccanica \\ e Aerospaziale \\ Rome, Italy \\ ivan.distefano@uniroma1.it
}

\author{
Paolo Cappuccio \\ Sapienza University of Rome, \\ Dipartimento di Ingegneria Meccanica \\ e Aerospaziale \\ Rome, Italy \\ paolo.cappuccio@uniroma1.it
}

\author{
Luciano Iess \\ Sapienza University of Rome, \\ Dipartimento di Ingegneria Meccanica \\ e Aerospaziale \\ Rome, Italy \\ luciano.iess@uniroma1.it
}

\begin{abstract}
The BepiColombo mission (ESA/JAXA) is devoted to the exploration of Mercury, the innermost planet of the Solar system. Among the experiments onboard of the spacecraft, the Mercury Orbit Radio Science Experiment (MORE) aims to gather information about the surface and the internal structure of the planet, and to carry out fundamental physics tests, thanks to a state-ofthe-art tracking system. During the cruise phase of BepiColombo, a general relativity test is performed in order to determine the Eddington parameter. Due to the proximity to the Sun, the solar irradiance random fluctuations have a dynamical effect that cannot be neglected. In this work a complete numerical simulation of the MORE fundamental physics experiment in cruise is carried out, taking into account the irradiance fluctuations. A precise orbit determination simulation has been used in order to assess the attainable accuracy on the determination of the Eddington parameter.
\end{abstract}

Keywords-Mercury, Radio science, Solar conjunction, Relativistic gravity, Solar irradiance

\section{INTRODUCTION}

The BepiColombo mission [1] is developed through a collaboration between the European Space Agency (ESA) and the Japan Aerospace eXploration Agency (JAXA); it was successfully launched on 20 October 2018 from the Centre Spatial Guyanais (Korou, French Guyana).

The Mercury Composite Spacecraft (MCS) is composed of two elements: the ESA's Mercury Planetary Orbiter (MPO) and the JAXA's Mercury Magnetospheric Orbiter (MMO). In addition to these, MCS comprehends the Mercury Transfer Module (MTM), which hosts the Solar Electric Propulsion System (SEPS), and the Sun shield. Reaching Mercury is really demanding in terms of mass of propellant, hence a combination of SEPS and flyby maneuvers will be exploited during the cruise phase, obtaining a weak capture into Hermean orbit in December 2025. The two spacecraft will be then released in two different orbits: the MMO will be inserted into an highly elliptical polar orbit $(590 \times 11600 \mathrm{~km}$ altitude), and it is devoted to explore the exosphere and the magnetosphere of the planet; the MPO will be placed in a lower near- circular, polar orbit (480 x $1500 \mathrm{~km}$ altitude), to investigate the surface and the deep interior of Mercury. The characteristics of its orbit permit to the MPO to improve the MESSENGER's result about the planet's rotation state, gravity field and tides. These ambitious objectives will be possible thanks to MORE advanced radio tracking technology [2]: the Ka-band Translator (KaT) together with the Deep Space Transponder (DST) provide a multifrequency radio-link in $\mathrm{X}$ and $\mathrm{Ka}$ bands ( 8 and $34 \mathrm{GHz}$ ). The three links $(\mathrm{X} / \mathrm{X}, \mathrm{X} / \mathrm{Ka}, \mathrm{Ka} / \mathrm{Ka})$ allow the complete cancellation of the dispersive noise, the main source of disturbance on radio tracking signals for interplanetary mission [3]. The end-toend link is expected to have an Allan deviation (frequency stability) of $10^{-14}$. This enables very accurate radiometric observables: the accuracy on Doppler measurements are smaller than $3 \mu \mathrm{m} / \mathrm{s}$ at an integration time of $1000 \mathrm{~s}$, and 20 $\mathrm{cm}$ in range measurements [4].

A general relativity test will be carried out both during the cruise phase, through a Superior Conjunction Experiment (SCE), and during the orbital phase. The SCE is performed through the estimation of the Parameterized Post Newtonian (PPN) parameter $\gamma$, which controls the deflection of a radiowave travelling from the spacecraft to the Earth. The best constraint on $\gamma$ has been obtained with the SCE of NASA's Cassini mission to Saturn in 2002, which estimated $\gamma=2.1 \pm 2.3 \times 10^{-5}[5]$. A comprehensive analysis of this experiment is given in [6], according to which this experiment could lead to a further improvement in the determination of this parameter, reaching an accuracy of $3 \times 10^{-6}$. The subject of this paper concerns the effect of the solar irradiance fluctuations on the results of the SCE. Random solar irradiance fluctuations range from 0.01 to $0.1 \%$ in timescales of a few days or hours [7]. For a mission such as BepiColombo, directed toward the innermost planet of the solar system, this is not a negligible effect. We verified through numerical simulations that a dynamical model which does not take these fluctuations into account, is not able to obtain reliable results: the trajectory retrieved from the orbit determination process would be significantly different from the real one. The tiny but continuous effect of solar radiation pressure variations is strongly correlated both with the state and $\gamma$, making the accuracy foreseen so far [6] on $\gamma$ optimistic.

The objective of this work is to describe a strategy able to absorb the effect of this disturbance, leading to a reliable result. Different strategies have been investigated in order to assess the effect of random fluctuations on the accuracy attainable on the determination of $\gamma$.

This work is organized as follows: in section II the physical effect exploited to obtain an estimate of the parameter $\gamma$ is described; then in section III the orbit determination method 
and the simulation setup are presented; in section IV some characteristics of the solar irradiance cycle are described; section $\mathrm{V}$ outlines the estimation strategy adopted to address the solar irradiance fluctuations problem; finally, results and conclusion are given in section VI.

\section{THE SHAPIRO EFFECT}

The first and most striking prevision of general relativity was the deflection of light coming from a star, due to the presence of a massive body in the path. The resulting increased path length causes a time delay: the signal passing near the massive body takes slightly longer to travel to a target and longer to return that it would if the mass was not present. This effect is known as Shapiro time delay; it is considered as the fourth test of general relativity, together with the deflection of light, the precession of the perihelion of Mercury and the gravitational redshift. In the PPN expansion of the Minkowsky metric, the amount of space-time curvature produced by a mass is controlled by the parameter $\gamma$. A signal exchanged between BepiColombo and an Earth-based antenna will undergo a time delay and a frequency shift [8], as expressed in (1) and (2):

$$
\begin{aligned}
& \Delta t=\left(\frac{G M}{c^{3}}\right)(1+\gamma) \ln \left(\frac{r_{1} r_{2}}{b^{2}}\right) \\
& \frac{d \Delta v}{v}=\frac{d \Delta t}{d t}=-2 \frac{(1+\gamma) G M}{c^{3} b} \frac{d b}{d t}
\end{aligned}
$$

where $\mathrm{G}$ is the gravitational constant, $\mathrm{c}$ is the velocity of light, $\mathrm{M}$ is the mass of the Sun, $r_{1}$ and $r_{2}$ are respectively the position of the transmitter and the receiver, in a reference system having the origin at the center of mass of the Sun; finally, $b<<r_{1}, r_{2}$ is the impact parameter, namely the distance of the closest approach of the radio wave to the center of the Sun (see Fig. 1). (1) and (2) are valid for small values of $b$. This effect is visible in the range and Doppler measurements exploited in orbit determination. Fig.1 shows the typical configuration of a superior solar conjunction (SSC). It is characterized by the fact that the Sun-Probe-Earth (SPE) angle is near 0 degree. This peculiar geometrical configuration of the three bodies involved (the Sun, the Earth and the spacecraft) enhances the signal path delay due to the space-time curvature [9]. During the interplanetary trajectory of BepiColombo, eleven SSC will take place, and each one is an opportunity to test general relativity estimating $\gamma$ with an orbit determination experiment.

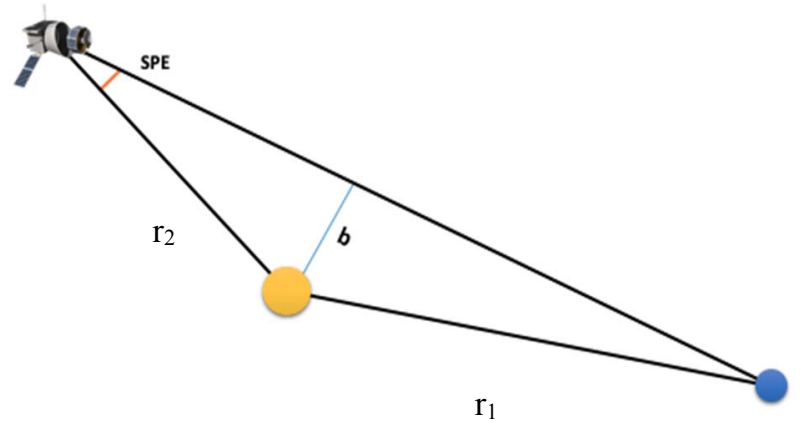

Fig. 1. Superior solar conjunction geometrical configuration.

\section{NUMERICAL SIMULATIONS}

\section{A. The method}

The orbit determination aims at obtaining the best estimate of the solve-for parameters that minimizes the discrepancies between real measurements (e.g. Doppler and range) and the predictions obtained by a mathematical model. In this work the classical pure batch orbit determination filter has been used. (3) shows the weighted least squares differential corrections with a priori information [10]:

$$
\delta \boldsymbol{x}_{e s t}=\left(H^{T} W H+W_{a p}\right)^{-1}\left(H^{T} W \delta \boldsymbol{y}+W_{a p} \delta \boldsymbol{x}_{a p}\right)
$$

where $\mathbf{x}$ is the n-dimensional vector of the differential correction for the estimated parameters, $\mathrm{H}$ is the design matrix, namely the matrix containing the partial derivatives of the observables quantities $\boldsymbol{y}$ with respect to the solved-for lists' elements, $\mathrm{W}$ is the weight matrix and $\delta \boldsymbol{x}_{a p}$ and $\mathrm{W}_{\mathrm{ap}}$ represent the a priori estimate and covariance matrix of $\mathbf{x}$. To simulate the MORE experiment, it is necessary to build a physical model which accurately describes BepiColombo cruise phase. This comprehends all the forces that act on the spacecraft, with particular emphasis on the solar irradiance realizations. To simulate the observed observables, synthetic observables have been generated according to the expected performance of the radio-tracking system.

\section{B. Simulation setup}

Eleven one-month observation arcs have been considered around the eleven SSC (as represented in Fig. 2). The tracking station used in this analysis is ESA's DSA 3 (Malargue, Argentina) because it is the only ESTRACK station capable to establish a three-frequency link in $\mathrm{X}$ and $\mathrm{Ka}$ band. The visibility conditions are considered satisfied when the spacecraft elevation above the ground station local horizon is $>10^{\circ}$. The multifrequency link permits a full plasma noise cancellation, but measurements taken when the signal path passes too close to the Solar corona [11] ( $b<7$ solar radii) are not reliable because the $\mathrm{X}$ band link enters in the strong scintillation regime, therefore these measurements have been ignored. The white Gaussian noise added to the generated data is based on the actual performances of the radio-tracking system: range and Doppler measurements are simulated every $300 \mathrm{~s}$ and $60 \mathrm{~s}$ with white noise of $30 \mathrm{~cm}$ and $12.25 \mu \mathrm{m} / \mathrm{s}$, respectively. The physical model considers the gravitational effect of all the planets and major bodies of the solar system; the gravity model takes into account relativistic effects. The shape of the spacecraft has been modeled as follows: a cylindrical bus with a cross-sectional area of $10 \mathrm{~m}^{2}$; the MPO solar array with an area of $8 \mathrm{~m}^{2}$; the two MTM solar arrays have both an area of $21 \mathrm{~m}^{2}$. The thermo-optical coefficients adopted have been measured on ground by the spacecraft prime contractor, Airbus. The attitude of the three solar panels is nearly fixed during one observation arc, with a total area exposed to the Sun of about $3 \mathrm{~m}^{2}$ for each MTM solar array. The MPO solar panel is considered not exposed to the Sun.

Solar irradiance fluctuations have been modeled through SOHO's measurements taken during the solar cycle 20032014, which provide one irradiance value per minute. It is worth noticing that for BepiColombo the attitude control is able to maintain the orientation with precision of $1^{\circ}$. Any variation of the solar radiation pressure acceleration due to 
uncertainties on attitude keeping is negligible, because this could lead to a perturbation of approximately $10^{-16} \mathrm{~km} / \mathrm{s}^{2}$, which cannot be spotted in any case.

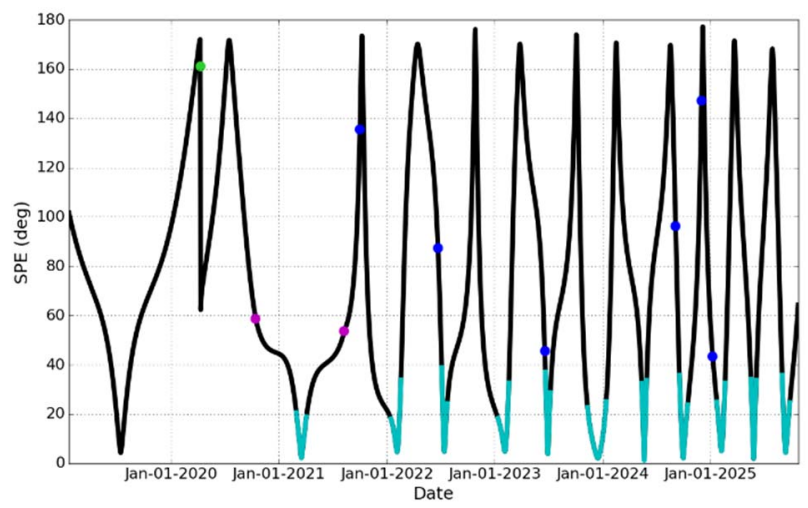

Fig.2. SPE angle variation during BepiColombo interplanetary trajectory. Black line represents the SPE angle during cruise and cyan line evidences the eleven observation arcs; green, purple and blue dots represent Earth, Venus and Mercury flybys, respectively.

\section{SOLAR IRRADIANCE FLUCTUATIONS}

The only non-gravitational acceleration taken into account in the equations of motion of the spacecraft is the solar radiation pressure. Its mathematical expression is given by:

$$
a_{s r p}=\frac{\Phi(t) f}{c m}
$$

where $\mathrm{c}$ is the speed of light, $\mathrm{m}$ is the mass of the spacecraft $(\mathrm{m}=2700 \mathrm{Kg})$, and $\boldsymbol{f}$ is a vector depending on the crosssectional area and the thermo-optical coefficients of the spacecraft surfaces exposed to the Sun. Finally, $\phi(t)$ is the solar flux, measured in $W / m^{2}$. The total solar irradiance (TSI) depends strongly on the distance from the Sun. It is possible to explicitly express this dependence as:

$$
\Phi(t)=\Phi_{1 A U}(t)\left(\frac{R_{E S}}{R}\right)^{2}
$$

Where $\Phi_{1 A U}(t)$ is the TSI measured at the distance of 1 Astronomical Unit (AU) from the Sun, and $R_{E S} / R$ is the ratio between the Earth-Sun distance (1 AU) and the spacecraft distance from the Sun. It is thence possible to notice and forecast how the solar irradiance varies with the distance from the Sun. What cannot be modelled is instead the temporal variation of $\Phi_{1 A U}(t)$ caused by the unpredictable solar activity: the variability of the TSI ranges between $0.01-0.1 \%$ in few days or hours, according to the solar conditions. The solar activity has a long quasi-periodical cycle of 11 years and several short period fluctuations which are still not completely understood. The two main sources of TSI variations are dark sunspots and bright faculae, two magnetic features appearing on the solar disk in different number and size. Both dark sunspot and bright faculae affect the solar emission respectively reducing and intensifying it: therefore, the observed fluctuations are the net effect of these two opposite phenomena [7]. According to Fig. 3, it is foreseeable that the randomly variable solar irradiance corresponding to the SCE period (with observation arcs spanning from 2021 to 2025), should be in the toughest part of its cycle (see Fig. 3 in the period from 2010 to 2014 , eleven years before the experiment).

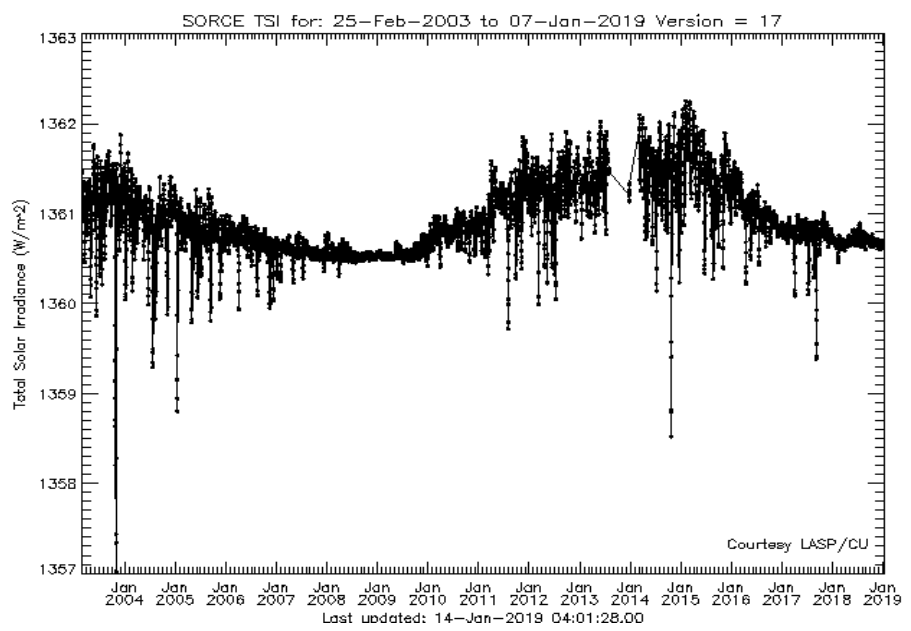

Fig. 3. Total solar irradiance fluctuations from 2004 to 2019 as measured by the space-based radiometer TIM (Total Irradiance Monitor) mounted on SORCE mission. Image courtesy of LASP CU.

\section{ESTIMATION STRATEGY}

The physical model used for the estimation process is the same as the simulation one, except for the fact that the solar irradiance variations are considered unknown. Measurements of solar irradiance are available through several space-based radiometers with noticeable accuracy; however, the solar irradiance is anisotropic, therefore the experiment cannot take advantages from TSI measurements collected from Earth's satellites, as MCS will lie in the opposite side of the Sun during SCE. As a priori information, the integrator considers a constant solar flux model with a reference value at 1 AU. This value is the mean value of the measurement of the arc, given the fact that although the fluctuations on the two side of the Sun will be different, it is reasonable to assume that the statistical properties will be analogous. In order to correct the discrepancy between the simulation and the estimation setup, a time-variable scale factor multiplying this constant solar flux reference value has been introduced as one of the solve-for parameters. The physical behavior of this scale factor allows it to be suitably described as a stochastic parameter. The aspect that needs to be addressed is the identification of the most appropriate noise model associated to the stochastic parameter: this will determine how the state and covariance for the stochastic parameter are affected by the process noise. In this work two noise types have been considered: a white noise model, given the unpredictability of these fluctuations, and an Exponentially Correlated Random Variable (ECRV) model, which requires to select a time constant $\tau$. Only one arc has been selected for these simulations, in order to be focused on the effect of solar irradiance fluctuation models rather than on the (already known) performances of each single arc. The estimated parameters are: the dynamical state of the spacecraft, the Eddington parameter $\gamma$, the thermo-optical coefficients of the spacecraft surfaces and the stochastic parameters representing the irradiance scale factors. This and the next sections are intended to show the effect of solar irradiance fluctuation models on the result of the experiment in a single SSC. The selected arc is the one 
corresponding to the third solar conjunction (July 2022), which is one of the best in terms of attainable uncertainty on $\gamma$ (thanks to a significant temporal variation of the impact parameter, see (2)). Different solar pressure realizations have been adopted in the data generation process, with the aim of assessing how the results on $\gamma$ accuracy varies according to the Sun activity in that period. Here we report the results corresponding to two solar irradiance models which represent two limit cases: an optimistic situation (case 1) in which the fluctuations are small and with an almost flat distribution (Fig. 6a), and a pessimistic case (case 2) in which the fluctuations are large (Fig. 6b).

\section{METHOD}

In both case 1 and case 2, the expected formal uncertainty on $\gamma$ has been determined according to an optimization process: the estimation of the stochastic scale factors requires to select the parameters which characterize the noise model. Firstly, in both cases the results show that a certain combination of parameters for an ECRV noise type yields a slightly better constraint on $\gamma$ than the white noise model. Selecting this kind of noise model means to describe the solar irradiance fluctuations as a process $\eta(t)$ which obeys the Langevin equation:

$$
\dot{\eta}(t)=-\beta \eta(t)+u(t)
$$

Where $\mathrm{u}(\mathrm{t})$ is white Gaussian noise with:

$$
\begin{gathered}
E(u)=0 \\
E[u(t) u(\tau)]=\sigma^{2} \delta(t-\tau)
\end{gathered}
$$

and $\beta=\frac{1}{\tau}$ where $\tau$ is the time constant (or correlation time). The stochastic part for the time update equations performed by the orbit determination filter is described by [12]:

$$
\eta_{j+1}=M_{j j} \eta_{j}+w_{j}
$$

where $\mathrm{M}$ is the transition matrix for the stochastic parameter and $w_{j}$ is the white process noise in discrete form for the stochastic parameter; in the case in which the $\mathrm{j}$ 'th parameter is using an ECRV noise model the following values are used:

$$
\begin{gathered}
M_{j j}=e^{-\frac{\Delta t}{\tau}} \\
Q_{j j}=\sigma_{s t}^{2}\left(1-e^{-\frac{2 \Delta t}{\tau}}\right)
\end{gathered}
$$

where $\mathrm{Q}$ is the offset covariance matrix; therefore, the ECRV noise model requires to select:

- The uncertainty of the stochastic parameter, $\sigma_{s t}$;

- $\quad$ The batch time duration for each scale factor, $\Delta t$;

- $\quad$ The time constant, $\tau$;

Fig.4 shows the autocorrelation of the solar irradiance measured by SORCE (Solar Radiation and Climate
Experiment) mission. As can be noted, a small peak is present at a correlation time near the solar rotation period (27.2753 d). Here we selected a time constant equal to the solar rotation cycle duration. Other values has been tested without a significant worsening of the result, but we choose to adopt this value which has a more physical significance and which minimizes the errors on the estimated parameters.

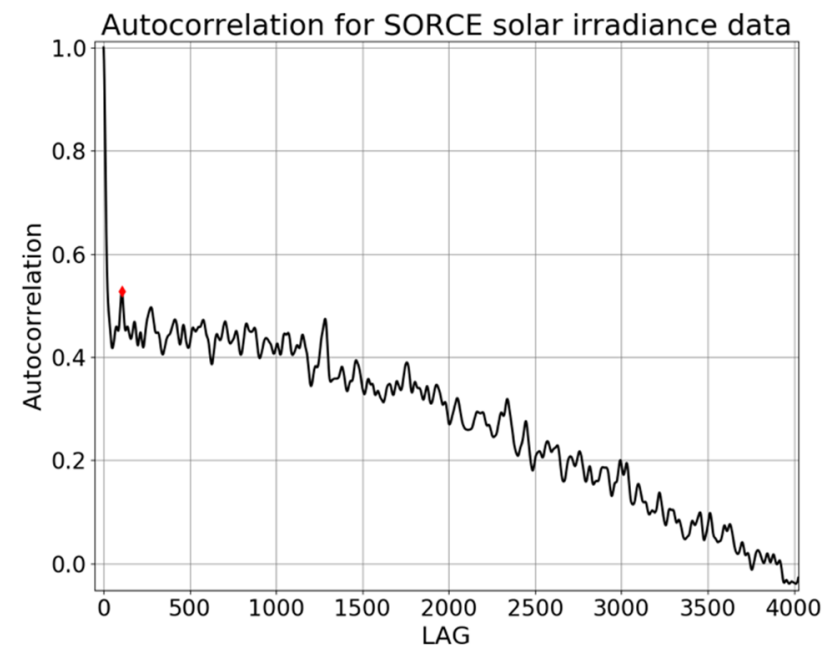

Fig. 4: Autocorrelation plot for solar irradiance data. The lag are represented by time batches of six hours. The red marker underlines that the first peak is after one solar rotation cycle ( 27 days).

The values of $\Delta \mathrm{t}$ and of $\sigma_{s t}$ instead have been picked on the basis of the quality of the orbit determination process' outcomes: when the choice of these parameters is inappropriate for the selected solar activity model, the true error on the estimated parameters increases reaching values which exceed 3-sigma. During the experiment, such a situation will be detectable thanks to the residuals between observed and computed observables (a clear indicator of eventual discrepancies between estimated dynamical model parameters and measurements): simulations' results confirm that in these cases the residuals distribution is not white and Gaussian, meaning that the estimated trajectory is different from the true one. This is an expected situation: the input $\sigma_{s t}$ delimitates the amount of displacement with respect to the starting constant value. This means that if $\sigma_{s t}$ is too tight, then the estimated oscillations will not compensate for the effect of the real ones and this effect would be attributed to other parameters; if instead $\sigma_{s t}$ is too large, then the effect of other parameters could be absorbed by the fluctuations. Furthermore, the choice of inappropriate stochastic input parameters often yields to unphysical results, attributing to the estimated thermo-optical coefficients negative values. Therefore, the numerical simulation of the experiment has been repeated with different values of $\Delta \mathrm{t}$ and $\sigma_{s t}$; analyzing the results for each pair of inputs on the basis of the observations pointed out above, we determined the optimal values to use for each simulated solar irradiance pattern. This pair should hence result in the smallest formal uncertainty on the estimate of $\gamma$ with a white Gaussian distribution of the residuals. Varying the values of $\Delta \mathrm{t}$ and $\sigma_{s t}$, it is possible to notice the influence on the estimate of $\gamma$ in terms of formal uncertainty $\sigma_{\gamma}$ and true error $\Delta_{\gamma}$ (see Fig.5). As introduced above, to retrieve the irradiance fluctuations by 
means of a stochastic model, $\sigma_{s t}$ has to be large enough according to their magnitude: this effect would be otherwise wrongly attributed to other dynamical parameters, hindering the possibility to reach convergence. Indeed, a larger $\sigma_{s t}$ corresponds to a lower true error $\Delta_{\gamma}$, but the cost is an increase of the formal uncertainty $\sigma_{\gamma}$ : of course this is in part due to the augmented dimension of the solve-for list (having more parameters to be estimated with the same amount of information). However, we attribute this effect mainly to the fact that the signal of the solar irradiance fluctuations shows to be highly correlated with the effect of having $\gamma-1 \neq 0$. Therefore the larger $\sigma_{s t}$ is, the easier would be to confuse the tiny relativistic signal with the similar but heavier one of the solar pressure variations. The growth of $\sigma_{\gamma}$ is thus an expression of this information, indicating the threshold under which the two effects cannot be separated. This explain why the flat oscillation case would be an optimistic situation, allowing to achieve a lower $\sigma_{\gamma}$. Also the choice of $\Delta \mathrm{t}$ influences the solution in terms of $\sigma_{\gamma}$ : if the selected batch duration is sufficiently below the one needed to represent important irradiance variations, the effect is an increased and unnecessary number of estimated parameters which would lead to a growth of $\sigma_{\gamma}$. However, a value of $\Delta \mathrm{t}$ overly large could risk to ignore some significant oscillations, yielding to an increase of the true error $\Delta_{\gamma}$ and signature on the residuals distribution.

The result of this analysis underlines that the key point to restrain the worsening of the experiment's outcome is the possibility to carry out this optimization process. The results indeed as outlined above, is strongly dependent on the input $\sigma_{s t}$ and $\Delta \mathrm{t}$. The choice of these parameters on the basis of the residuals distribution can also be supported by the estimated solar pressure variations; the results show that these oscillations can be reconstructed with good approximation (see Fig. 6), at least at the level needed to differentiate between strong or weak solar activity. The standard deviation of the estimated solar pressure variations could give more confidence on the selection of the input parameters for the stochastic model (as shown in Fig. 7).

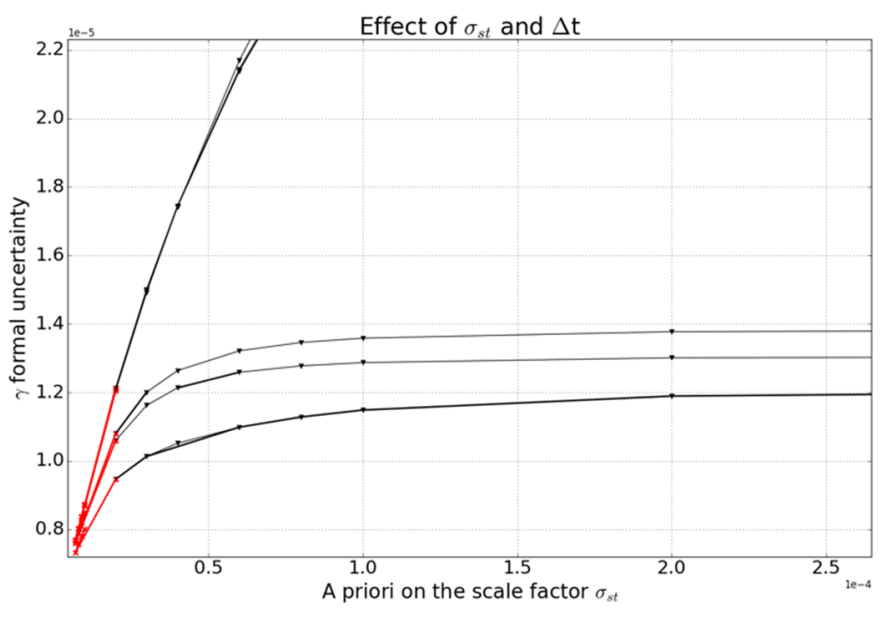

Fig. 5: Formal uncertainties on the determination of $\gamma$ varying the value of $\Delta t$ and $\sigma_{\text {st }}$ for flat irradiance fluctuations (case 1): the red crosses represent the solution which true error exceed the 3- $\sigma$ limit or when the convergence criteria are not met; the black points are the acceptable solutions. The higher curves correspond to $\Delta t$ of 48,24,3 and 6 hours, while the lower ones corresponds to $\Delta t$ of $84,72,96$ hours.

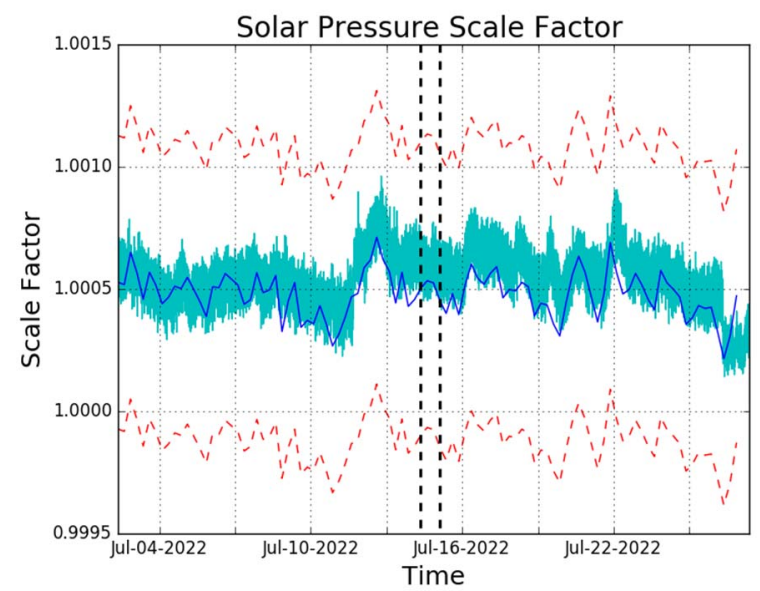

(a)

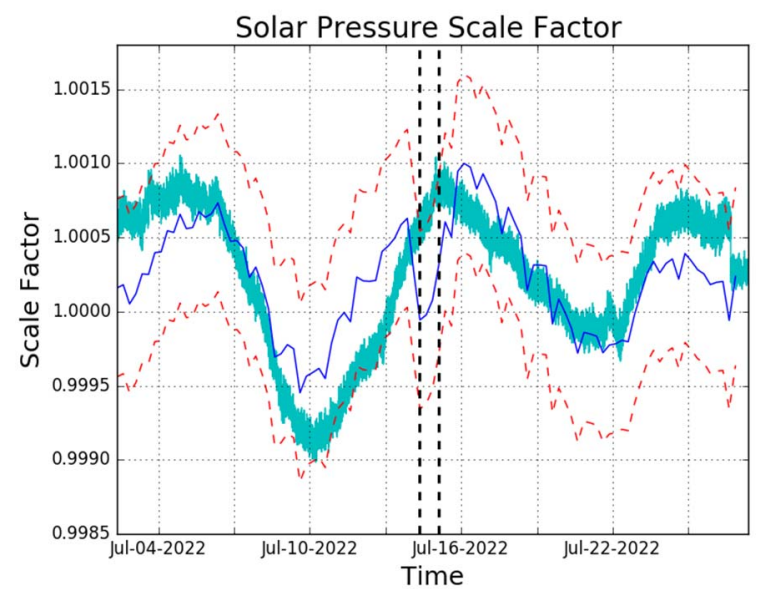

(b)

Fig. 6: Solar irradiance scale factors: case 1, flat distribution (a), case 2, strong fluctuations (b). Cyan line represents the one-minute simulated solar irradiance scale factors; blue line indicates the estimated values; red dashed lines are the 3-sigma uncertainties; black dashed lines limit interval of ignored measurements $(b<7$ solar radii).

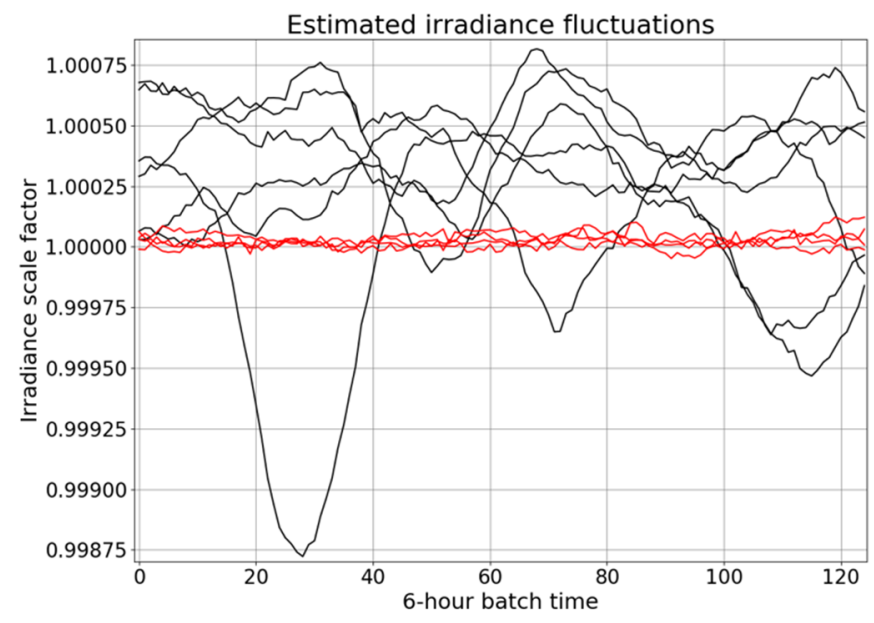

Fig. 7: Estimated solar irradiance fluctuations varying the simulated model. The black lines represent cases in which the optimization process indicates $\sigma_{s t}=2 \times 10^{-4}$ while the red lines represent cases in which the best $\sigma_{\text {st }}$ indicated is $2 \times 10^{-5}$. 


\section{RESULTS}

The simulation of the third SSC, considering a mismodeling of the solar radiation pressure force due to irradiance fluctuations, shows an increase of the uncertainty on the determination of $\gamma$ with respect to the results presented in [6] for both the two cases presented in the preceding section. In case 1 , the best attainable result would be $\sigma_{\gamma}=1 \times 10^{-5}$, an increment of a factor of two with respect to the attainable result in the same arc if irradiance fluctuations were completely known $\left(\sim 6 \times 10^{-6}\right)$; in case 2 , which is a worstcase scenario, the deterioration factor would be even larger.

The analysis has been repeated with a large set of solar irradiance models, employing the data from eleven years of total solar irradiance measurements of SORCE mission. Spanning over the results of this extended range of simulations, the potential contribute of the conjunction has been considered as the average of the outcomes ( $\sigma_{\gamma}=$ $2.44 \times 10^{-5}$ for SSC 3). Then, for each one of the eleven observation arcs, the same procedure has been carried out. According to this strategy, a combined solution which would exploit each one of the eleven SSC would lead to the final result of $\sigma_{\gamma}=7.5 \times 10^{-6}$, which is $\sim 2.2$ times the result of [6]. We underline that the solution are chosen between the ones which present a true error $\Delta_{\gamma}$ which is below the $1-\sigma_{\gamma}$ level, in order to maintain a conservative approach, making this result more reliable.

\section{CONCLUSION}

In this work we analyzed the effect of solar irradiance fluctuations on the SCE of BepiColombo, assessing the need to address this problem and describing a strategy to mitigate the worsening of the solution. We simulated the orbit determination experiment including in the solve-for list some additional dedicated parameters, which represent a stochastic scale factor introduced to describe the irradiance variation. We have shown that to estimate these scale factors the noise model has to be cautiously constructed, and we determined how it would be possible to build this stochastic model obtaining a reliable solution for the determination of $\gamma$, which still represents a significant improvement with respect to previous estimate.

A decrease of the total area exposed to the Sun in case 1 would act as a meaningful contribute to further ameliorate the solution.

Furthermore, it has to be assessed if the Italian Spring Accelerometer (ISA) could be used to detect the accelerations induced by solar radiation pressure fluctuations. This would be of outermost importance because, in principle, it would be possible to obtain the same performances of [6].

\section{REFERENCES}

[1] Benkhoff J, Casteren J V, Hayakawa H, Fujimoto M, Laakso H, Novara M, Ferri P, Middleton H R and Ziethe R 2010 BepiColombocomprehensive exploration of mercury: mission overview and science goals Planet. Space Sci. 58 2-20 (Comprehensive Science Investigations of Mercury: The scientific goals of the joint ESA/JAXA mission BepiColombo)

[2] Iess L and Boscagli G 2001 Advanced radio science instrumentation for the mission BepiColombo to mercury Planet. Space Sci. 491597 608

[3] Tortora P, Iess L and Ekelund J E 2002 Accurate navigation of deep space probes using multifrequency links: the Cassini breakthrough during solar conjunction experiments IAF Abstracts, 34th COSPAR Scientific Assembly The Second World Space Congress, Houston, TX, USA vol. 1 p 675

[4] Iess L, Asmar S and Tortora P 2009 MORE: an advanced tracking experiment for the exploration of mercury with the mission BepiColombo Acta Astronaut. 65 666-75

[5] Bertotti B, Iess L and Tortora P 2003 A test of general relativity using radio links with the Cassini spacecraft Nature 425 374-6

[6] Imperi, L., Iess, L., 2017. The determination of the post-Newtonian parameter $\gamma$ during the cruise phase of BepiColombo. Classical Quant. Gravity 34 (7), 075002. URL: http://stacks.iop.org/0264$9381 / 34 / \mathrm{i}=7 / \mathrm{a}=075002$.

[7] Magnitudes and timescales of total solar irradiance variability, Greg Kopp, J. Space Weather Space Clim., 6 (2016) A30, Published online: 2016-07-25, DOI: https://doi.org/10.1051/swsc/2016025

[8] Shapiro I I 1964 Fourth test of general relativity Phys. Rev. Lett. 13 789-91

[9] Will CM 1993 Theory and Experiment in Gravitational Physics (Cambridge: Cambridge University Press)

[10] Tapley, B., Schutz, B., Born, G., 2004. Statistical Orbit Determination. Elsevier Science.

[11] Tortora P, Iess L, Bordi J J, Ekelund J E and Roth D C 2004 Precise Cassini navigation during solar conjunctions through multifrequency plasma calibrations J. Guid. Control Dyn. 27 251-7

[12] Tapley, B., Schutz, B., Born, G., 2004. Statistical Orbit Determination. Elsevier Science. 